Revue d'histoire de l'Amérique française

ZAS REVUE D.HISTOIRE DE L'AMÉRIQUE FRANÇAISE

\title{
Entre ville et campagnes, les subdélégués de l'intendance canadienne (1675-1763)
}

\section{Sébastien Didier}

Volume 70, numéro 1-2, été-automne 2016

URI : https://id.erudit.org/iderudit/1038291ar

DOI : https://doi.org/10.7202/1038291ar

Aller au sommaire du numéro

Éditeur(s)

Institut d'histoire de l’Amérique française

ISSN

0035-2357 (imprimé)

1492-1383 (numérique)

Découvrir la revue

Citer cet article

Didier, S. (2016). Entre ville et campagnes, les subdélégués de l'intendance canadienne (1675-1763). Revue d'histoire de l'Amérique française, 70(1-2), 113-137. https://doi.org/10.7202/1038291ar
Résumé de l'article

De 1675 à 1763, la colonie de Nouvelle-France est administrée par un intendant qui délègue dans les villes une partie de ses pouvoirs à des subdélégués. Ayant toute sa confiance et commis pour l'informer et exécuter ses ordres sur « toutes sortes d'affaire ", ils agissent aussi bien en ville qu'à la campagne, diffusant l'autorité et la justice de l'intendant dans toute la province. Plus que la domination des notables urbains en milieu rural, nos travaux montrent à quel point le réseau de l'intendant s'adapte aux localités, notamment par le recrutement des subdélégués. 


\title{
Entre ville et campagnes, les subdélégués de l'intendance canadienne ( $\left(6_{75}-\mathrm{I} 763\right)$
}

\author{
SÉBASTIEN DiDIER \\ Université Rennes 2 \\ Université de Montréal
}

\begin{abstract}
RÉSUMÉ • De 1675 à 1763, la colonie de Nouvelle-France est administrée par un intendant qui délègue dans les villes une partie de ses pouvoirs à des subdélégués. Ayant toute sa confiance et commis pour l'informer et exécuter ses ordres sur «toutes sortes d'affaire», ils agissent aussi bien en ville qu'à la campagne, diffusant l'autorité et la justice de l'intendant dans toute la province. Plus que la domination des notables urbains en milieu rural, nos travaux montrent à quel point le réseau de l'intendant s'adapte aux localités, notamment par le recrutement des subdélégués.
\end{abstract}

ABSTRACT - Between 1675 and 1763, to assist in the colony's governance, the intendant of New France was appointing sub-delegates in the towns. He delegated part of his powers to these officers whom he trusted to inform him and execute his orders concerning "all sorts of affairs". Deployed over the entire territory, urban and rural, these sub-delegates extended the intendant's authority and jurisdiction throughout the province. Our research shows that, in addition to a dominance of urban notables in rural areas, the intendant's administrative strategies were adapted to local circumstances, notably through the recruitment of sub-delegates.

\section{T es subdélégués de l'intendance canadienne sont-ils des administra- teurs urbains ou ruraux ${ }^{1}$ ? La question n'est pas anodine. Elle mène à}

1. Cet article développe une communication du $68^{\mathrm{e}}$ Congrès de l'Institut de l'histoire de l'Amérique française à Montréal, le 16 octobre 2015. Il s’inspire donc de sa thématique : “Urbanités ». 
penser l'administration coloniale de la France d'Ancien Régime sous un nouvel angle.

Les subdélégués canadiens n'ont, en effet, jamais fait l'objet d'étude historique approfondie ${ }^{2}$. Certains travaux sur l'administration leur consacrent quelques lignes, un paragraphe, ou plus rarement un chapitre ${ }^{3}$. Le Dictionnaire biographique du Canada en présente un grand nombre, mais évoque peu leur subdélégation ${ }^{4}$. La dispersion des sources entre les registres de l'intendance de Québec, les différents fonds des juridictions royales canadiennes et les Archives françaises d'outre-mer à Aix-en-Provence a longtemps dissuadé de toute recherche conséquente sur le sujet. Heureusement, les travaux de numérisation et d'indexation permettent à présent de regrouper les sources dispersées et de mieux connaître les subdélégués.

L'intendance de Nouvelle-France n'est pas inconnue. Les travaux de Marcel Trudel, par exemple, décrivent sa mise en place dans les années $1663-1670^{5}$. Elle se construit en même temps que certaines intendances métropolitaines. Mais elle reprend les pouvoirs confiés aux compagnies de commerce, se rattache exclusivement au ministère de la Marine et s'organise par la suite en fonction de l'ordonnance de la Marine de 1681. Les études qui la considèrent prennent principalement le point de vue des intendants. Les biographies de Claude-Thomas Dupuy par Jean-Claude Dubé et celle de François Bigot par Guy Frégault l'illustrent bien ${ }^{6}$. Plus récemment, la thèse de Marie-Ève Ouellet propose de l'aborder à travers

2. Mis à part le mémoire de master dont découle cette contribution: Sébastien Didier, Les subdélégués des intendances de Bretagne et de Nouvelle-France, Antrain, Fougères, Hédé et Montréal, une étude prosopographique comparée, mémoire de master (histoire), Université Rennes 2, 2011. Sur la subdélégation en France: Colette Brossault, Les intendants de Franche-Comté, 1674-1790 (Paris, La Boutique de l'Histoire, 1999); Julien Ricommard, "Les subdélégués des intendants aux XVII ${ }^{\mathrm{e}}$ et XVIII ${ }^{\mathrm{e}}$ siècles », Information historique, (1962), 139-148, 190-195 et (1963), 1-7.

3. Gonzalve Doutre, et Edmond Lareau, Le droit civil canadien, vol. I., Histoire générale du droit canadien (Montréal, A. Doutre, 1872), 133 ; Gustave Lanctôt, L'administration de la Nouvelle-France (Montréal, Éd. du Jour, 1971), 74; Édouard-Zotique Massicotte, «Les tribunaux et les officiers de justice de Montréal», Bulletin des recherches historiques, 37, 5, (1931), 311-312; Jean-Claude Dubé, Claude-Thomas Dupuy, intendant de la Nouvelle-France, 1678-1738 (Montréal, Fides, 1969), 121-122; Louise Dechêne, Le peuple, l'État et la guerre au Canada sous le Régime français (Montréal, Boréal, 2008). Jean-Philippe Garneau, Justice et règlement des conflits dans le gouvernement de Montréal à la fin du Régime français, mémoire de maîtrise (histoire), Université du Québec à Montréal, 1995 : 60-92.

4. Par exemple: Donald J. Horton, "Michel de Villebois de La Rouvillière, Honoré», Dictionnaire biographique du Canada en ligne, www.biographi.ca/fr [consulté le 29 décembre 2009].

5. Marcel Trudel, Histoire de la Nouvelle-France, vol. IV, La seigneurie de la Compagnie des Indes occidentales, 1663-1674, (Montréal, Fides, 1996).

6. J.-C. Dubé, Claude-Thomas Dupuy..., op. cit.; Guy Frégault, François Bigot, administrateur français (Montréal, Les Étude de l’Institut d’Histoire de l’Amérique Française, 1948), 2 vol. 
l'analyse de la procédure administrative ${ }^{7}$. Son analyse diplomatique des archives et particulièrement des ordonnances fait ressortir l'importance de la culture judiciaire dans la pratique et la rhétorique de l'intendance. Pour mieux comprendre cette pratique et «dissoudre [comme le préconise Catherine Desbarats,] les abstractions que sont l'État et la société par une patiente identification des acteurs multiples ", il convient de s'intéresser aussi aux hommes de l'intendant: les subdélégués ${ }^{8}$.

La question posée ici propose donc de changer de point de vue. Elle aborde l'intendance par le bas de la hiérarchie, par ses agents les plus proches des administrés. La seule échelle provinciale ne suffit plus. L'uniformité administrative apparente éclate face aux analyses locales. D’ailleurs, aucune subdélégation canadienne ne se ressemble.

La Nouvelle-France est vaste et protéiforme ${ }^{9}$. Dans les faits, elle ne dépend pas entièrement de l'intendant. Les deux subdélégations atlantiques de Plaisance en Terre-Neuve et de Port-Royal en Acadie, puis de Louisbourg sur l'île-Royale (île-du-Cap-Breton) et de Port-La-Joie sur l'Île Saint-Jean (Île-du-Prince-Édouard) correspondent directement avec le ministre. Elles ne nous intéresseront pas ici. L'intendant s'occupe essentiellement de la vallée laurentienne. De Pabos en Gaspésie à Détroit dans les Grands Lacs, cela représente déjà un espace long de plus de $2000 \mathrm{~km}$. Cette distance l'oblige à déléguer. Dans les années 1680, il crée trois subdélégations dans les villes de Montréal, Trois-Rivières et Québec. Les subdélégués se déploient donc dans la province à partir des villes. Dotés des pouvoirs de l'intendant, ils le représentent dans ces chefs-lieux, mais aussi et surtout auprès des ruraux.

La question de l'urbanité renforce la multiscalarité de l'analyse en ajoutant un niveau. Elle dévoile surtout un particularisme canadien. En métropole, beaucoup de chefs-lieux de subdélégations sont de simples bourgs ${ }^{10}$. Aucun ne s'inscrit dans des circonscriptions aussi vastes. La

7. Marie-Ève Ouellet, "Et ferez justice. " Le métier d'intendant au Canada et dans les généralités de Bretagne et de Tours au 18 siècle (1700-1750), thèse de doctorat (histoire), Université de Montréal et Université Rennes 2, 2014.

8. Catherine Desbarats. «La question de l'État en Nouvelle-France », dans Philippe Joutard et Thomas Wien, dir., Mémoires de la Nouvelle-France (Rennes, France, Presses universitaires de Rennes, 2005), 195.

9. Catherine Desbarats et Allan Greer, "Où est la Nouvelle-France?», Revue d'histoire de l'Amérique française, 64, 3-4 (2011): 31-62.

10. Guy Arbellot et Jean-Pierre Goubert, dir., Carte des généralités, subdélégations et élections en France à la veille de la Révolution de 1789 (Paris, CNRS, 1986); Yann Lagadec, «Penser l'espace administratif pour le moderniser, L'exemple des subdélégations en Bretagne au XVIII siècle », dans Brigitte Caulier et Yvan Rousseau, dir., Temps, espaces et modernités, mélanges offerts à Serge Courville et Normand Séguin (Québec, Les Presses de l’Université Laval, 2009), 311-321. 
dualité entre ville et campagne semble beaucoup plus imposante dans la structure du territoire administratif canadien.

Cette question appelle finalement à une analyse complète comprenant la subdélégation à la fois comme circonscription en examinant son territoire, comme fonction en décrivant ses prérogatives et comme représentation en s'intéressant aux subdélégués eux-mêmes.

\section{LE DÉPLOIEMENT DES SUBDÉLÉGUÉS DES VILLES VERS LES CAMPAGNES}

L'histoire institutionnelle interroge rarement la création du territoire administratif de l'Ancien régime. Elle révèle pourtant les pratiques et les stratégies du pouvoir. L'intendance canadienne s'appuie sur trois pôles urbains particulièrement structurants.

\section{La création du territoire administratif autour des villes}

Qu'elle soit urbaine ou rurale, la subdélégation a pour but premier le déploiement du pouvoir et des services de l'intendance sur son territoire. Elle doit donc, en théorie, s'adapter aux caractéristiques de ce dernier. La Nouvelle-France est construite autour du Saint-Laurent. Ce grand fleuve en est la colonne vertébrale, mais aussi la première porte d'entrée et le principal moyen de communication interne. Le territoire est lui-même structuré par trois villes fondées sur son cours à des endroits stratégiques, faciles à défendre et permettant des communications avec les Autochtones pour la traite des fourrures. Ce sont Québec en 1608, Trois-Rivières en 1634 et Ville-Marie, future Montréal, en 1642. Le faible peuplement de ces centres peut laisser dubitatif quant à leur urbanité. Rappelons brièvement, qu'hier comme aujourd'hui, l'urbain ne se définit pas par un seuil de peuplement ${ }^{11}$. En Nouvelle-France, Québec, Montréal et Trois-Rivières sont bien des espaces pérennes de concentration et de diversité à la fois du bâti, de la population, des fonctions et des échanges. Ils abritent environ un cinquième de la population pendant toute la période française. Même Trois-Rivières, dont la population ne dépasse pas les 600 habitants avant la Conquête, structure l'espace canadien par une polarisation et un centralisme certains. Elle concentre entre 1 et $2 \%$ de la population canadienne et 10 à $20 \%$ de celle de son aire d'influence. Sur ce dernier espace, elle exerce un pouvoir à la fois religieux, politique et administratif. Elle

11. Claude Nières, «La petite ville du XVII ${ }^{\mathrm{e}}$ siècle au milieu du XIX ${ }^{\mathrm{e}}$ siècle», dans Jean-Pierre Poussou et Philippe Lopes, Les petites villes du Moyen âge à nos jours (Bordeaux, CNRS-Presses universitaires de Bordeaux, 1987), 499-503. 
obtient petit à petit de nombreux attributs urbains: une juridiction en 1657 devenue royale en 1680, une communauté religieuse pourvoyant à l'enseignement et à la santé dès 1697, un marché hebdomadaire officiel en 1722. Surtout, Trois-Rivières est une ville parce qu'elle est reconnue comme telle, dès la fin des années 1660 , par ses habitants et ses visiteurs ${ }^{12}$. Elle offre aux Trifluviens une identité urbaine.

Dans ces trois nouvelles villes laurentiennes, les débouchés commerciaux attirent la population. Dès les années 1640, le gouverneur Charles Huault de Montmagny y place des lieutenants pour assurer la défense des habitants et des places marchandes ${ }^{13}$. Les trois gouvernements particuliers de la Nouvelle-France sont nés. Ils désignent d’abord un mandat pour superviser la gestion des forts et des redoutes, le maintien d'une garnison et l'entretien d'une milice. Puis, au fur et à mesure que leurs responsabilités s'étoffent, le gouvernement devient une circonscription territoriale. Comme en témoigne une commission pour le commandement militaire de Québec en l'absence du gouverneur Frontenac, les frontières géographiques des trois gouvernements particuliers semblent fixées dès le début des années $1670^{14}$. Celui de Montréal semble d'abord ne concerner que l'île. Puis, en 1685, un mémoire du gouverneur Denonville l'étend jusqu'à la rivière Richelieu ${ }^{15}$.

Traditionnellement, l'historiographie canadienne considère que les limites des gouvernements correspondent aux points de jonction des trois foyers de peuplement en expansion. Toutefois, au début des années 1690, l'aire démographique trifluvienne n'a toujours pas joint celle de Montréal ${ }^{16}$. Certes presque toutes les rives du Saint-Laurent sont déjà concédées en seigneuries, mais les frontières des gouvernements se sont d'abord appuyées sur des points de défense ou de contrôle aisé du fleuve, avant de chercher

12. Bibliothèque et Archives nationales du Québec (BAnQ), E1,S4,SS2,P229-287, Louis-Armand de Lom d'Arce de La Hontan, Voyages du baron de La Hontan dans l'Amérique septentrionale, t.1 (Amsterdam, L’Honoré, 1705), 24; Claude-Charles Le Roy de La Potherie, “Un mémoire de Le Roy de La Potherie sur la Nouvelle-France adressé à M. de Pontchartrain, 1701-1702», Bulletin des recherches historiques, 22, 7, (1916), 218; Julie Marleau, La juridiction royale des Trois-Rivières en Nouvelle-France: la ville, les officiers et les habitants (1663-1760), mémoire de maîtrise (histoire), Université de Sherbrooke, 2014.

13. Marie-Claire Daveluy, "Chomedey de Maisonneuve, Paul de », Dictionnaire biographique du Canada en ligne [consulté le 10 juin 2016]; Douville, Raymond, "Champflour, François de», Dictionnaire biographique du Canada en ligne, www.biographi.ca/ fr [consulté le 29 décembre 2009].

14. Pierre-Georges Roy, Ordonnances, commissions des gouverneurs et intendants de la Nouvelle-France, 1639-1706, vol. 1 (Beauceville, L’Eclaireur Limitée, 1924), 44.

15. Archives nationales françaises d'outre-mer (ANOM), Col., C11A, vol. 7, fol. 127-129.

16. Richard Colebrook Harris, The Seigneurial system in early Canada: a geographical study (Madison, Milwaukee, Londres et Québec, University of Wisconsin Press et Les Presses de l'Université Laval, 1968), 92-93. 
à correspondre avec la trame seigneuriale encore mouvante en cette fin du XVII ${ }^{e}$ siècle. Celle qui sépare le gouvernement de Trois-Rivières et de Québec se situe dans une zone difficilement navigable du fleuve coudé par la Pointe-Platon. Passé Trois-Rivières, le Saint-Laurent s'élargit pour former le lac Saint-Pierre. Il devient indéfendable avant le chapelet d'une douzaine d'îles qui forme la frontière avec le gouvernement de Montréal.

Ce partage militaire de la province entre les deux gouverneurs particuliers et le gouverneur général est constitutif de la géographie administrative canadienne de la fin du XVII ${ }^{e}$ siècle à 1792. Il sert de base à l'intendant qui adopte les mêmes limites pour ses subdélégués. Les subdélégations canadiennes s'appuient donc d'abord sur les trois villes les plus structurantes du Canada, puis s'étendent aussi à leur campagne environnante.

\section{La formation des subdélégations depuis les centres urbains}

La mention la plus ancienne d'un éventuel subdélégué canadien date de 1669. Avant de reprendre les fonctions d'intendant au Canada, Jean Talon pose ses conditions au ministre. Entre autres, il demande «que le s[ieur] Talon de Villeneuve, son cousin germain, [...] ait la commission de subdélégué pour agir à Québec tandis que Talon se transportera dans les habitations, et les postes avancés ${ }^{17}$ ». Le faible nombre d'habitants ne nécessite pas encore une administration très puissante. En dehors de Québec, il peut donc se contenter de visites annuelles. Parfois, son subdélégué québécois se déplace. En 1677, René-Louis Chartier quitte la capitale pour poursuivre une enquête judiciaire à Montréal ${ }^{18}$. Mais dès le début des années 1680 , cette situation n'est plus tenable. Montréal s'est considérablement développée et l'intendance doit y contrôler le commerce des fourrures. Aussi, le bailli est-il «subdélégué par Monsieur l'Intendant pour informer des contrevenans aux ordres du Roy sur le fait du commerce du castor avec les Anglois ${ }^{19}$ ”.

Basé à Montréal, Migeon de Branssat n'est toutefois pas soumis à une quelconque limite géographique. En 1685, il exerce même ses fonctions à Québec. Nicolas Dupont de Neuville, «nommé par Monsieur l'Intendant pour faire ses fonctions en son absence en qualité de son subdélégué», s'insurge, considérant que le Montréalais empiète sur ses prérogatives. Ce différend finit devant le Conseil souverain qui reconnaît les

17. ANOM, Col., C11A, vol. 3, fol. 49.

18. «Documents inédits: Inventaire des biens de René-Louis Chartier, sieur de Lotbinière (Québec, 13-17 juin 1701)", Revue d'histoire de l'Amérique française, 21, 4 (1968): 822.

19. Arrêt du 8 novembre 1683, cité dans La législature de la Province de Québec, Jugements et délibérations du Conseil souverain de la Nouvelle-France (Québec, Imprimerie A. Côté et Cie, 1886), 1040-1042. 
droits de Migeon ${ }^{20}$. La même année, l'intendant de Meulles reçoit l'autorisation de la part du ministre, d'« establir à Montréal, un subdélégué pour faire ces fonctions dans la partie méridionale de Canada ${ }^{21}$ ». Il s'agit d'un premier pas vers la territorialisation des subdélégations canadiennes qui concerne aussi le subdélégué de Trois-Rivières récemment nommé22.

En cette fin du XVII ${ }^{e}$ siècle, les subdélégations de Québec, Trois-Rivières et Montréal deviennent de véritables circonscriptions. Elles reprennent les frontières des trois gouvernements qui découpent le Canada. La première mention de cette association date d'avril 1689 pour Québec ${ }^{23}$. Pour les deux autres subdélégations, les ressorts sont probablement définis en même temps. Mais les sources les font apparaître plus tard dans des commissions datées de 1709 et 1711 . Les subdélégations comprennent donc désormais une grande portion d'espace rural en voie de colonisation.

\section{La croissance des subdélégations}

Le cadre géographique des gouvernements particuliers est très étendu pour des subdélégations. Celui de Montréal s'étale sur plus de $130 \mathrm{~km}$ le long du fleuve. Celui de Trois-Rivières sur une centaine de kilomètres. Celui de Québec est encore plus imposant, avec $200 \mathrm{~km}$ sur la rive nord et près de 400 sur la rive sud. Nous ne parlerons pas de superficie. En effet, l'écoumène canadien longe le fleuve sans s'étendre dans les terres. D'ailleurs, la population des subdélégations est dérisoire. En 1692, d’après le recensement de l'intendance, le gouvernement de Montréal compte 3484 habitants dont 800 à Ville-Marie même. Celui de Québec compte 7426 habitants dont 1570 à Québec et celui de Trois-Rivières, 1521 habitants dont 300 dans la ville ${ }^{24}$. Cependant, la population canadienne croît constamment. Elle triple avant la Conquête en 1760. Depuis 1680, presque chaque année, au moins une nouvelle paroisse est créée le long du Saint-Laurent ${ }^{25}$. Le gouvernement de Montréal comprend ainsi seulement 7 paroisses en 1674, puis 20 en 1713 et 45 avant la Conquête anglaise. Le gouvernement de Trois-Rivières voit l'ouverture de 14 paroisses de 1674 à 1760, celui de Québec celle de 43

20. Ibid.

21. ANOM, Col. C11A, vol. 7, fol. 191.

22. BAnQ,Vieux-Montréal, Fonds du notaire Séverin Ameau, microfilm n ${ }^{\circ} 3742,24$ février 1688, Minutes du notaire Séverin Ameau, 24 février 1688.

23. Bibliothèque et Archives Canada (BAC), MG 18-H64.

24. Statistique Canada. Recensement de la Nouvelle-France, E-STAT. http: / / estat2.statcan.gc.ca/cgi-win/ cnsmcgi.exe?Lang=F\&EST-Fi=EStat $\backslash$ Francais $\backslash$ SC_RR-fra.htm [consulté le 20 mai 2010].

25. Serge Courville et Normand Seguin, dir., Atlas historique du Québec. La paroisse (Sainte-Foy, Les Presses de l’Université Laval, 2001), 16. 
paroisses $^{26}$. Le cadre géographique apparemment stable des subdélégations cache la réalité d'un territoire en perpétuelle croissance. En effet, l'étendue de l'occupation sur les rives du Saint-Laurent donne une meilleure image du territoire réellement concerné par la subdélégation.

\section{Le déploiement des subdélégués autour des centres urbains}

Comme l'écoumène se prolonge et se densifie, l'intendant et ses trois subdélégués ne suffisent pas toujours au service. À Montréal, le subdélégué, également commissaire de la Marine, est rapidement débordé pendant la guerre de succession d'Espagne. À partir de 1709, l’intendant Raudot doit donc recourir à un second subdélégué spécialisé dans la justice: Pierre Raimbault. Plus tard, en 1727, alors qu'il fait confiance à ce dernier pour être le seul subdélégué montréalais, l'intendant Dupuy nomme deux subdélégués à Québec: Théodore Denis de Vitré et Pierre André de Leigne. Son successeur, Gilles Hocquart, ne nomme qu'un seul subdélégué par ville. Mais rapidement, il doit multiplier ses représentants. En 1736, il quitte la province pendant huit mois pour appuyer devant le Roi une demande d'aide financière. Il nomme alors un deuxième subdélégué dans le gouvernement de Québec. Or cette situation perdure finalement jusqu’à la Conquête. Surtout, Gilles Hocquart crée deux nouvelles subdélégations avec la nomination en 1737 de Georges Lefebvre de Bellefeuille à Pabos en Gaspésie et celle de Robert Navarre à Détroit en $1743^{27}$.

Ces subdélégations périphériques n’ont probablement jamais eu de ressort délimité. Leur isolement géographique les en dispense. Toutefois, elles témoignent de la croissance de l'espace administratif et de l'influence de l'intendance. Cette influence s'amplifie aussi à une dizaine de kilomètres au nord-ouest de Trois-Rivières. En 1741, un nouveau subdélégué prend en charge les forges de Saint-Maurice ${ }^{28}$. Sans surcharger de travail le subdélégué de Trois-Rivières, l'intendant prend le contrôle de cette industrie jugée indispensable pour produire munitions, canons et poêles à chauffer. Le choix du subdélégué spécialisé ne se fait pas au hasard. Guillaume Estèbe est choisi, probablement parce qu'il est le garde-magasin du Roi à Québec et connaît bien les stocks et besoins de l'armée. L'intendant envoie aussi une commission au père Charles Barbel à Rimouski en $1736^{29}$. Mais il est difficile de savoir s'il s'agit d'une subdélégation pérenne ou ponctuelle.

26. André La Rose, «Les registres paroissiaux au Québec avant 1800 », Études et recherches archivistiques, 2, (1980): 53 .

27. BAnQ, Québec, E1, S1, P2933, 3499.

28. BAnQ, Québec, E1, S1, P3366.

29. BAnQ, Québec, E1, S1, P2831. 
En effet, Gilles Hocquart utilise régulièrement des subdélégués temporaires. Ils agissent souvent le temps d'une simple mission et permettent de suppléer les subdélégués permanents en leur évitant un long déplacement à la campagne. Ces subdélégués sont nommés parmi les notables ruraux. Ce sont les curés de Contrecœur en 1737 et de l'Ange-Gardien en 1738, deux paroisses éloignées de $60 \mathrm{~km}$ de Montréal $^{30}$. Dans le gouvernement de TroisRivières en 1741, la subdélégation ponctuelle du procureur fiscal de SaintPierre-les-Becquets évite un voyage de $50 \mathrm{~km}$ au subdélégué Godefroy de Tonnancour ${ }^{31}$. Du côté de Québec, Gilles Hocquart octroie de telles subdélégations au curé, au notaire ou au capitaine de milice de Beauport et Grondines en 1738, de Saint-Anne-de-Beaupré en 1736 et 1743, mais aussi de la Rivière-Ouelle en 1740 et du Cap-Saint-Ignace en $1747^{32}$. Il semble faire le plus usage de ces commissions ponctuelles.

Mais il n'est pas le premier. Jacques Raudot envoie ainsi le sieur Haimard à Beauport en $1707^{33}$. Il utilise même ces commissions en ville, commettant François Hazeur pour juger un contentieux entre deux marchands de Québec ${ }^{34}$. Cela lui vaut de vives critiques adressées au ministre Pontchartrain par le procureur général Ruette d'Auteuil. Ce dernier donne une liste d'une dizaine de noms de subdélégués devant lesquels l'intendant renvoie les parties «quand il n'a pas le loisir de les entendre ou qu'il ne veut pas s'en donner la peine $[\ldots]$ et ainsi prive les juges de leurs fonctions ${ }^{35}$ ». La plainte du procureur général n’aboutit pas et le Roi le révoque en 1707.

Michel Bégon, l'intendant suivant, utilise aussi les commissions temporaires mais les octroie le plus souvent au procureur général du Conseil souverain, désormais Mathieu-Benoît Collet. Malgré ce témoignage, les subdélégations ponctuelles sont rares et limitent peu les affaires rurales des subdélégués permanents. Les sources de la subdélégation canadienne, minces et souvent indirectes, ne permettent aucune statistique significative mais simplement un aperçu très global. Sur 174 actes ou preuves d'actes, 70 , soit $40 \%$, concernent les centres urbains, le reste concerne la ruralité canadienne. Ces traces d'une action réelle du subdélégué en ville

30. BAnQ, Québec, E1, S1, P2927; J.-P. Garneau, Justice et règlement..., op. cit., 86.

31. BAnQ, Québec, TL5, D2009-48.

32. BAnQ, Québec, E1, S1, P3048, 3061, 3189, 3763; Assemblée législative du Canada, Arrêts et règlements du Conseil supérieur de Québec et ordonnances et jugement des intendants du Canada (Québec, E. R. Fréchette, 1855), 215 et 370. J.-P. Garneau, Justice et règlement..., op. cit., 86.

33. Complément des ordonnances et jugements des seigneurs et intendants du Canada, précédédes commissions des dits gouverneurs et intendants et des différents officiers civils et de justice (Québec, E. R. Fréchette, 1856), 128-129.

34. BAnQ, Québec, TL5, D393.

35. ANOM, Col C11A 34/fol.445-450. 
et à la campagne sont assez régulières sur toute la période. Leur cartographie montre la couverture par les subdélégués de l'ensemble de leur gouvernement.

Le recours à la subdélégation temporaire n'est pas systématique et ressemble plus à un palliatif qu'à une véritable solution. Il témoigne avant tout du refus de créer plus de subdélégations rurales. En métropole, généralement, le surcroît de travail est géré en nommant de nouveaux subdélégués par scission des circonscriptions concernées. L’intendant de Nouvelle-France préfère, lui, diviser les tâches en nommant des subdélégués secondaires ponctuels ou spécialisés. Ce recours est la preuve de la force structurante des villes laurentiennes dans le déploiement de l'intendance. Cette force est étonnante lorsqu' on connaît bien les prérogatives essentiellement rurales des subdélégués.

\section{LES PRÉROGATIVES DES SUBDÉLÉGUÉS ET LEUR POUVOIR SUR LES RURAUX}

Les prérogatives des subdélégués sont avant tout décrites par des sources prescriptives. Mais quelques actes permettent d'en appréhender la mise en pratique. Nous découvrons alors des subdélégués avec une forte emprise sur la police rurale et la justice.

\section{La justice des affaires sommaires}

Selon les commissions nommant les subdélégués canadiens, leur première prérogative semble être judiciaire. Dès 1689, la commission de René-Louis Chartier lui donne comme première fonction d' "ouïr les plaintes des peuples et habitants du district de Québec, exercer et administrer la justice ${ }^{36}{ }^{\prime}$. Cependant, la justice des subdélégués devient véritablement importante à partir de 1706. L'intendant Jacques Raudot tend alors à laisser l'administration classique de l'intendance à son fils pour s'occuper de la justice ${ }^{37}$. Les subdélégués sont sollicités et exercent ce pouvoir jusqu'à l'invasion de 1760 . Ces procès peuvent concerner des contentieux entre marchands ou artisans comme en 1708, quand Pierre Raimbault juge le conflit opposant les maçons Gilbert Maillet et Pierre Couturier pour le marché public de la prison de Montréal ${ }^{38}$. Le même Pierre Raimbault s'est vu attribuer par l'intendant l'affaire opposant à Lachine une fille de cultivateur au curé à

36. BAC, MG18-H64.

37. D. J. Horton, «Raudot, Jacques», Dictionnaire biographique du Canada en ligne, www.biographi.ca/ fr [consulté le 29 décembre 2009].

38. BAnQ, Vieux-Montréal, TL4, S1, D1115. 
propos de ses grains ${ }^{39}$. En 1743 et 1744, le subdélégué de Québec Henri Hiché condamne des habitants du Château-Richer et de La Pointe-Lévy à partager les frais de clôture de leurs terres ${ }^{40}$.

Les subdélégués jugent beaucoup de conflits de succession. En fait, la justice des subdélégués canadiens concerne toutes les affaires, rurales ou urbaines, "qui ne sont pas d'une assez grande conséquence pour aller devant les juges ${ }^{41} »$. En 1729 et 1738, les commissions de subdélégués limitent leur pouvoir aux différends qui n'excédent pas la somme de 100 livres pour le subdélégué de Québec, 500 pour le Montréalais et 200 pour le Trifluvien ${ }^{42}$. Jean-Philippe Garneau rappelle les articles 1 à 5 du titre XVII de l'Ordonnance civile de 1667, selon lesquels tombent sous l'appellation d'affaires sommaires les affaires concernant la police, les biens domestiques, les loyers, les animaux en divagation, les gages de serviteurs et honoraires professionnels et «tout ce qui requiert célérité $[. .$.$] pourvu$ que ce qui sera demandé n'excède la somme ou valeur de mille livres ${ }^{43}$ ». Le plus important à retenir est répété dans les commissions et instructions données aux subdélégués: le subdélégué doit avant tout se faire le juge des cas «qui demandent d'être jugés sommairement et sans frais ${ }^{44}$ " pour "éviter [aux habitants] des longueurs de procédures ${ }^{45}$ " et des dépenses importantes. La justice des subdélégués canadiens s'apparente à celle des juges de paix créée en métropole à la Révolution. Elle répond au besoin judiciaire dont témoigne l'importance de l'infra-justice au XVIII ${ }^{\mathrm{e}}$ siècle ${ }^{46}$. Elle apporte donc autant à la ville qu'à la campagne.

\section{La lutte contre la contrebande avec les commis du Domaine}

Les subdélégués sont également souvent sollicités par l'intendant pour régler des affaires concernant le Domaine du Roi, et donc juger les fraudes économiques liées au monopole de la traite des fourrures. Cette justice spéciale touche elle aussi autant les ruraux que les urbains. En effet, si les marchands sont souvent urbains, les coureurs des bois viennent régulièrement des paroisses rurales. Les arrêts et ordonnances royales interdisant «à toutes personnes de transporter en quelque manière que ce fut, même par l'entremise des Sauvages, aucuns castors dans les colonies angloises,

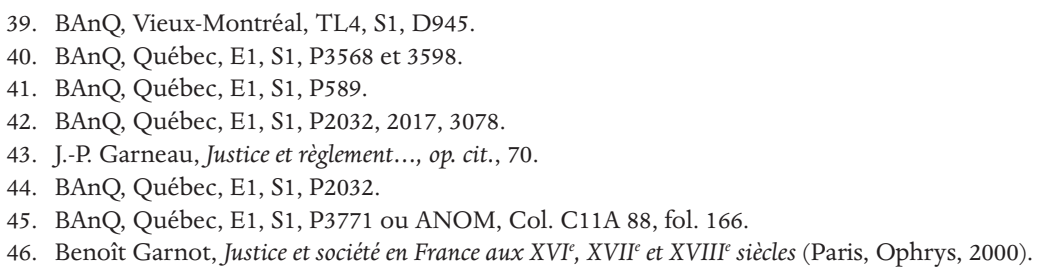


et à ses sujets en Canada de vendre ni tenir dans leurs magasins aucunes marchandises ni effets venant des colonies angloises ", se répètent en 1707, 1709,1715 et $1719^{47}$.

Dans ce combat contre la fraude, les subdélégués montréalais comptent beaucoup. Le premier, Jean-Baptiste Migeon de Branssat, n'est d'abord commis, rappelons-le, que "pour informer contre ceux qui entreprendrons les dits voïages de traite, les faire arrêter et constituer prisonniers et les envoïer sous bonne et sûr garde en cette ville de Québek pour leur procès ${ }^{48}$ ». Une lettre de l'intendant Champigny au ministre semble indiquer qu'à la bonne saison, le subdélégué, à l’image de Louis Tantouin de La Touche, se déplace à Lachine ou «au haut de la colonie pour y visitter les canots qui montoient à Missillimakinak» et autres postes de traite ${ }^{49}$. Cette inspection permet de vérifier les licences et les marchandises emportées. Au retour des canots, les commis du Domaine d'Occident saisissent les marchandises des contrebandiers, puis réclament la certification de leurs procès-verbaux par les services de l'intendance.

En 1715, un procès-verbal est ainsi adressé à Clairambault d'Aigremont par Nicolas Lanoullier, agent de Néret et Gayot, au sujet des écarlatines (étoffes de laine) anglaises saisies chez Mme de La Pipardière ${ }^{50}$. Dans les années 1715-1726, Pierre Raimbault, subdélégué spécialisé dans la justice, est particulièrement actif contre la fraude. Pour lui faciliter la tâche, il reçoit, en 1722, une commission lui permettant d' "accompagner les commis des directeurs du Domaine d'Occident et du castor dans toutes les visites qu' ils feront dans les maisons et autres lieux [..., de] les répéter en leurs procèsverbaux et recevoir leurs affirmations sur les saisies ${ }^{51}$ ». Le jugement des contrebandiers relève ensuite de la prérogative exclusive de l'intendant ou de ses subdélégués, «Sa Majesté en interdisant la connoissance à tous autres juges» en $1707^{52}$. De ces procès, nous connaissons, entre autres, celui des habitants de La-Prairie-de-la-Madeleine qui se sont absentés sous prétexte d'aller en chasse et sont allés à Orange dans des canots de "sauvages ${ }^{53}$ ".

47. Assemblée législative du Canada, Édits, ordonnances royaux, déclarations et arrêts du Conseil d'État du Roi, concernant le Canada (Québec, E. R. Fréchette, 1854), 347-348.

48. BAC, F3, vol. 6, fol. 25, citée dans Pauline Dubé, La Nouvelle-France sous Joseph-Antoine Lefebvre de La Barre, 1682-1685 (Sillery, Septentrion, 1993), 45-46.

49. ANOM, Col., C11A, vol. 16, fol.88.

50. ANOM, Col., C11A, vol. 35, fol. 235-236.

51. BAnQ, Québec, E1, S1, P1405.

52. Arrêt du Conseil d’État du 25 juin 1707, cité dans Édits, ordonnances royaux..., op. cit., 302-304.

53. BAnQ, Québec, E1, S1, P1465. 


\section{La police rurale avec les capitaines de côte}

"Enjoignons aux lieutenants-généraux et nos subdélégués des villes de Québec, Montréal et Trois-Rivières, de tenir la main à l'exécution de la présente ordonnance et de la faire publier et afficher partout où besoin sera, à ce qu'aucun n'en ignore ${ }^{54}$.» Cette phrase se retrouve dans la plupart des ordonnances de l'intendant. Par contre, quand il s'agit de police urbaine, soit de l'établissement d'un marché sur la place d'armes en 1706, ou encore de la défense de laisser vaquer les cochons dans les rues en 1710, elle ne s'adresse qu'au lieutenant général ${ }^{55}$. Seule la surveillance des cabarets regarde les subdélégués dans la police urbaine. Les ordonnances de l'intendant et les commissions des subdélégués donnent pouvoir aux subdélégués d'autoriser la tenue des cabarets et de surveiller la vente d'alcool, pour éviter notamment son accès aux Amérindiens ${ }^{56}$. Une seule ordonnance du subdélégué Dupont de Neuville nous permet de confirmer son usage véritable, mais elle regarde les cabarets ruraux ${ }^{57}$. Or, de ce côté, pas de doute, la police des côtes est le domaine exclusif des subdélégués. L'intendant doit d'ailleurs mettre les choses au clair en 1743 : les capitaines de côte dépendent de lui et de ses subdélégués pour tout ce qui regarde la police et jamais du lieutenant général ${ }^{58}$. Le gouverneur non plus ne peut s'adresser à eux sans son accord ${ }^{59}$.

Dans les faits, la police rurale est du ressort des subdélégués canadiens depuis la fin du XVII siècle. Mais cette fonction n'est sanctionnée par les commissions de subdélégués qu’à partir de 1726. Tombent sous leur responsabilité les questions «des districts de paroisses, de la construction ou de la réfection des chemins et ouvrages publics» et tous les litiges fon$\operatorname{ciers}^{60}$. Ils doivent aussi faire respecter la coutume seigneuriale. En 1708, le séminaire de Saint-Sulpice, seigneur de l'île de Montréal, demande à Pierre Raimbault d'agir contre les habitants de la côte Saint-Sulpice et de La Rivière-de-l'Assomption qui coupent impunément du bois sur ses terres. Le subdélégué rédige alors une ordonnance condamnant les contrevenants à 10 livres d'amende applicables par l'économe du séminaire ${ }^{61}$. Une ordon-

54. Ordonnance du 5 décembre 1705, citée dans Arrêts et règlements du Conseil supérieur..., op. cit., 257-258.

55. Arrêts et règlements du Conseil supérieur..., op. cit., 261, 265 et 276.

56. BAnQ, Québec, E1, S1, P1777, 2826, 3790.

57. BAnQ, Québec, R1, P38.

58. ANOM, C11A, vol. 79, fol. 140-141.

59. ANOM, C11A, vol. 89, fol. 138-141.

60. BAnQ, Québec, E1, S1, P1940.

61. Archives du Séminaire de Saint-Sulpice à Montréal, P1:2-80, tiroir 7, n 80 . 
nance similaire est produite en 1689 pour le séminaire de Québec victime de la chasse au loup marin sur les îles de sa seigneurie de Beaupré ${ }^{2}$.

Surtout, les subdélégués doivent veiller aux intérêts du Roi et au bon ordre dans les campagnes. Ils agissent alors avec l'appui des capitaines de côte qui forment une sorte de relais supplémentaire au pouvoir royal. De cette manière, l'ordonnance du 18 octobre 1758 organise le logement des troupes à la campagne ${ }^{63}$. Les subdélégués s'occupent d'établir le rôle du logement. Ils le transmettent au capitaine de milice pour exécution. En cas de contestation des soldats ou des habitants ou de conflits entre eux, capitaines de milice et commandants de compagnie doivent en référer au subdélégué. La même méthode est adoptée pour la répartition de la corvée, notamment pour la voirie.

En 1775, François-Joseph Cugnet l'explique clairement. Les subdélégués doivent s'assurer de la praticabilité des chemins saison après saison. Plusieurs fois par an, les chemins doivent être «entretenus et réparés par chaque habitans sur la devanture des terres, desquelles ils sont tracés ${ }^{64} »$. Lorsque la neige recouvre le pays, les routes sont battues et balisées par des épinettes. Le travail le plus ennuyeux arrive au printemps quand la fonte des neiges détruit les chemins. Il faut souvent recreuser les fossés et poser des planches dans les endroits boueux. Aux abords des chemins, les habitants doivent également clôturer leur propriété pour éviter la divagation du bétail. Aidé par les capitaines de milice, le subdélégué est le garant de la bonne application de ces règlements. En 1685, le gouverneur prévient le subdélégué Migeon du manque d'entretien du chemin entre Ville-Marie et le poste des "sauvages» de la Montagne. Celui-ci ordonne aussitôt le défrichage et le nettoyage de la voie sous peine d'expropriation $^{65}$. En 1715, le lieutenant général de la juridiction de Montréal enregistre l'établissement du chemin royal entre la ville et la côte SaintMichel $^{66}$. L'acte décrit la construction du chemin sous les ordres de Charles Alavoine, capitaine de la côte Saint-Michel, agissant au nom des seigneurs de l'île et du subdélégué de l'intendant. Le lien avec les capitaines de côte est indispensable mais ne suffit pas. Pour l'efficacité de la corvée, du logement des troupes, et également du recrutement de la milice, l'intendance

62. Centre de référence de l’Amérique française à Québec, Séminaire 35, n $23 \mathrm{c}$.

63. Henri-Raymond Casgrain, dir., Lettres et pièces militaires : instructions, ordres mémoires, plans de campagne et de défense 1756-1760 (Québec, Impr. de L.-J. Demers, 1891), 54-59.

64. François-Joseph Cugnet, Traité de la police (Québec, Guillaume Brown, 1775).

65. P.-G. Roy, Ordonnances, commission..., op. cit., 129-130.

66. BAnQ, Vieux-Montréal, TL4, S1, D1657. 
et ses subdélégués doivent bien connaître la population et sa répartition.

\section{Les recensements avec les seigneurs}

Les subdélégués ont un rôle d'informateur et participent aux recensements de la population rurale avec l'aide des seigneurs. Certes, selon Richard Lalou et Mario Boleda, «bien que les recensements semblent être devenus, au fil des années, une opération de routine, on ne sait que peu de chose sur la méthode employée ${ }^{67}$ », mais quelques indices peuvent être relevés. S’appuyant sur des déclarations des curés et seigneurs, le recensement de 1685 se présente comme un tableau donnant pour chaque seigneurie le nombre d'églises, moulins, hommes, femmes, garçons, filles, armes, terres, bétail et ecclésiastiques ${ }^{68}$. La division en gouvernement apparaît avec le recensement de $1692^{69}$. Les subdélégués y sont probablement pour quelque chose. Un échange des intendants avec le ministre semble le confirmer en 1709. Les intendants Raudot s'excusent de ne pouvoir envoyer le recensement en incriminant le retard des seigneurs. Le ministre se voit donc proposer

d'envoyer aux s[ieu]rs Raudot un ordre de Sa M[ajes]té pour obliger tous les seigneurs hauts-justiciers, ou censeurs de cette colonie de remettre dans le mois de may, année par année, à leurs subdélégués dans les trois villes de ce pays, le recensement des seigneuries qu'ils ont dans le Gouvernement de chaque ville, à peine de 2001 [ivres] t[ournois] d'amende ${ }^{70}$.

Les trois subdélégués semblent donc s'occuper de la centralisation, voire de la compilation des résultats. Le recensement de l'année 1721 fait exception. Cette année-là, le Roi demande la restructuration des districts paroissiaux. Le sieur Collet, procureur général du Conseil supérieur, est alors "commis et subdélégué» pour relever les habitants de la colonie avec des déclarations " tant sur le nombre de leur familles que sur l'étendue de leur concession et de ce quelles contiennent ${ }^{71}$. Du 4 février au 3 juin 1721, il parcourt donc les rives du Saint-Laurent dans le but final d'élaborer les 82 districts de paroisses du règlement du 3 mars $1722^{72}$. Mais, rappelons-le, le

67. Richard Lalou et Mario Boleda, «Une source en friche: les dénombrements sous le régime français", Revue d'histoire de l'Amérique française, 42, 1, (1988): 50.

68. ANOM, Col., G1 vol. 461, fol. 3.

69. Ibid., fol. 6.

70. ANOM, Col., C11A, vol. 30, fol. 304.

71. BAnQ, Québec, E1, S1, P1347.

72. André Morel, "Collet, Mathieu-Benoît», Dictionnaire biographique..., op. cit. Voir aussi David Dubois, Les procès-verbaux sur la commodité et l'incommodité des districts paroissiaux de Mathieu Benoît Collet (1721), mémoire de maîtrise (histoire), Université Laval, 2003. 
territoire canadien évolue en même temps que sa population et ces districts doivent régulièrement être revus par l'intendance et ses subdélégués.

\section{La fixation des districts et chemins ruraux avec les habitants}

Chaque subdélégué doit régulièrement intervenir dans les paroisses pour reconfigurer leurs limites. Des conflits et tensions apparaissent fréquemment pour savoir à quelle paroisse se rattachent les nouvelles familles et leurs propriétés. En 1725, Pierre Raimbault reçoit un ordre de l'intendant pour fixer les districts de La Prairie-de-la-Madeleine et de Saint-Ours. Il doit «se transporter sur les lieux pour entendre les curés seigneurs et habitants, à leurs frais et dépens, tant des $\mathrm{d}$ [ites] paroisses que des paroisses voisines et interessées au règlement des districts et de tout en dresser les procès verbaux ${ }^{73}$ ». En 1734, les problèmes sont tels que le grand-voyer Lanoullier de Boisclerc entreprend de visiter une par une les paroisses de Nouvelle-France pour redessiner les districts. L'intendant lui donne exceptionnellement une commission de subdélégué pour qu'il puisse, en plus, régler les différends «entre les habitants, soit pour closture mitoyenne, fossés de ligne, escoulement des eaux, dégâts et dommages causés par les bestiaux et animaux, et autres matières sommaires personnelles ${ }^{74}$ ». Habituellement, ces conflits sont réglés par ordonnances du subdélégué et les frais de déplacement sont à la charge des requérants ${ }^{75}$. En découlent des procès-verbaux de chaînage conservés en nombre dans les greffes d'arpenteurs ${ }^{76}$. Une sorte de cadastre rural se crée ainsi, parfois lors de conflits interpersonnels, d'autres fois par une refonte totale, souvent lors d'oppositions intercommunautaires. Le subdélégué intervient donc souvent face à des assemblées d'habitants pour jouer le rôle d'arbitre.

Le même processus se met en place pour la création de la voirie. Le subdélégué assemble les paroissiens concernés afin de décider du meilleur tracé. Louis Lavallée décrit ces assemblées où chaque particulier défend ses intérêts ${ }^{77}$. En 1722, dans la salle presbytérale de La-Prairie-de-laMadeleine, s'opposent les paroissiens pour ou contre la construction de deux ponts sur les rivières Saint-Jacques et de la Tortue. Les uns soutiennent le projet pour circuler plus vite et pouvoir se rendre plus souvent à la messe. Les autres refusent de participer puisqu'ils iront toujours plus

73. BAnQ, Québec, E1, S1, P1690.

74. BAnQ, Vieux-Montréal, TL4, S35, P28.

75. BAnQ, Vieux-Montréal, TL4, S1, D3721.

76. BAnQ, Vieux-Montréal, CA301, S2, P539, 544, 742; S43, P28-2

77. Louis Lavallée, La Prairie en Nouvelle-France: étude d'histoire sociale (Montréal, McGill-Queen's University Press, 1992), 167-169. 
vite en canot. Dans le cas d'un chemin, chacun privilégie un tracé à la fois proche de son habitation et hors de ses terres. Le subdélégué ne peut rien imposer mais chaque fois, c'est à lui de trancher. Une ordonnance d'Honoré Michel de Villebois de La Rouvillière témoigne du nombre d'acteurs différents mobilisés pour l'élaboration d'un chemin.

En 1726, une partie des habitants de la côte de Saint-Léonard demande le tracé d'un chemin de leur paroisse à la ville de Montréal. L'arpenteur Auger propose une ligne droite entre les terres de trois propriétaires. Ce chemin «est sans contredit le plus convenable ayant esté réglé et assigné de l'agrément et consentement de $\mathrm{M}^{\mathrm{rs}}$ les seigneurs, [...] M. Becquancour pour lors grand voyer, [et] les principaux habitans assemblés ${ }^{78}$ ». Le subdélégué, Jean-Baptiste de Silly, ordonne donc les travaux. Malgré ses efforts, certains habitants et «mutins» refusent de participer à la corvée. Les travaux traînent. Les séditieux sont poursuivis. Un nouveau projet est validé, quelque temps avant l'été 1731, par Jean-Eustache Lanoullier de Boisclerc, le nouveau grand voyer. Les "principaux habitans de la coste» en appellent aussitôt au subdélégué et Honoré Michel bloque ce projet décidé, selon lui, «au mépris de la demande des plus notables et du plus grand nombre des habitans [...], de $\mathrm{M}^{\mathrm{rs}}$ les seigneurs, du s[ieu]r curé [...] et de l'utilité publique, [...] sans faire attention aux ordonnances» des subdélégués successifs. Avec l'aide du capitaine de côte, il suspend l'exécution du chemin. Très instructif, ce document nous présente deux groupes de pression assez dissuasifs pour bloquer deux projets, un grand voyer distant et un subdélégué chef d'orchestre, s'appuyant sur les notables locaux.

Les subdélégués exercent donc une influence importante sur les acteurs et les territoires ruraux. Avec leur pouvoir de police et de justice, ils font respecter les ordonnances royales. Leurs prérogatives les amènent aussi à organiser le territoire rural de leur circonscription. Cependant, loin d'imposer un plan, ils le façonnent en s'adaptant aux réalités et aux acteurs locaux. Ils observent alors le même pragmatisme que l'intendant lors de leur recrutement.

\section{LES SUBDÉLÉGuÉS, DES NOTABLES À L'IMAGE DE LEUR CHEF-LIEU}

Malgré des prérogatives communes, les subdélégués de l'intendance canadienne sont d'origines très variées. Tous exercent en même temps d'autres fonctions plus rémunératrices et l'intendant les recrute parmi les

78. Archives nationales de France, Col. $\mathrm{F}^{3}$, fol. 348-349, d’après la transcription dactylographiées de l’Univers Culturel de Saint-Sulpice à Montréal, P1 : 6.46-1302a. 
notables locaux les plus aptes. Or, ces hommes sont très différents d'un chef-lieu à l'autre.

\section{À Montréal, des commissaires de la Marine en carrière}

Montréal est une ville très militarisée du fait de la proximité de la frontière avec la Nouvelle-Angleterre et l'Iroquoisie. À partir de 1686, l'intendant n’y choisit pas vraiment ses subdélégués. Il nomme presque automatiquement le commissaire ordonnateur de la Marine. Administrateur royal le plus puissant de la colonie, juste après le gouverneur et l'intendant, il fait le lien entre le militaire et le gestionnaire. Concrètement, il est chargé des dépenses et de la logistique militaire. Il connaît bien son département puisqu'il y contrôle les troupes et la distribution des armes et des vivres dans les magasins du Roi. Par contre, en 1709 pendant la guerre de succession d'Espagne et en 1757 pendant celle de Sept Ans, l'intendant doit le suppléer en commettant des subdélégués spécialisés dans la justice: Pierre Raimbault, puis Jacques Joseph Guiton. Surtout, le commissaire de la Marine change souvent. Neuf subdélégués se succèdent de 1682 à 1760 demeurant huit ans en moyenne. Ce fort renouvellement est souvent une caractéristique des subdélégations urbaines.

À Montréal, particulièrement, le commissaire subdélégué ne cherche pas à s'installer. À part le dernier, Pierre-Michel Martel, aucun n'est natif de la province. Ils considèrent leur poste canadien comme une étape vers des commissariats plus prestigieux comme ceux de Bordeaux ou Rochefort. Même si beaucoup d'entre eux se marient au Canada, leur avenir et leurs terres sont en métropole. Ils acquièrent très peu de biens fonciers canadiens et n'apparaissent quasiment pas dans les minutes notariales. Célibataire, François Clairambault ne loue même pas de maison. Il vit chez le gouverneur puis dans une annexe de ses bureaux ${ }^{79}$. Honoré Michel habite dans les magasins du Roi jusqu'à son mariage. Son contrat stipule alors que la mariée obtienne de son grand-père maternel la moitié de la grande maison familiale à colombage ${ }^{80}$. Cette situation ne traduit aucune pauvreté. Dans les années 1740, Honoré Michel et son successeur Jean-Victor Varin emploient au moins deux domestiques et possèdent tous les deux un esclave noir ${ }^{81}$. Jean-

79. BAnQ, Vieux-Montréal, Fonds du notaire Antoine Adhémar, microfilm n ${ }^{\circ} 642,25$ mai 1709. Voir aussi fonds du notaire Jean-Claude Louet, microfilm $\mathrm{n}^{\circ} 10880,24$ décembre 1728 .

80. BAnQ, Vieux-Montréal, Fonds du notaire Nicolas-Auguste Guillet de Chaumont, microfilm $\mathrm{n}^{\circ} 2106,13$ novembre 1737.

81. ANOM, Col. C11A, vol. 89, fol. 9, vol.72, fol. 86. Fiche $\mathrm{n}^{\circ} 152174$ et $\mathrm{n}^{\circ} 293952$ du « Programme de recherche en démographie historique de l'Université de Montréal» (PRDH), [en ligne], Montréal, Université de Montréal, 1999-2006, www.genealogie.umontreal.ca. 
Victor Varin réaménage la maison qu'il loue pour y organiser régulièrement des bals accueillant la haute société montréalaise. Dans sa correspondance, Mme Bégon la décrit avec ses cheminées en plâtre moulé et sa table en fer à cheval de 20 couverts $^{82}$.

Le retour en métropole se présente à tous comme un but de carrière. Les commissaires de la Marine subdélégués réclament chacun leur tour à l'intendant et au ministre un congé pour la France. Mais il s'agit pour eux de gérer leur patrimoine et peser dans des négociations pour obtenir un autre poste ${ }^{83}$. À peine arrivé, Mathieu Gaillard et Louis Tantouin de La Touche obtiennent le poste de commissaire à Rochefort. Certains ne rentrent pas au bon moment ou n'arrivent pas à obtenir un poste métropolitain. Ils sont renvoyés dans d'autres colonies. Jean-Baptiste Silly devient commissaire de la marine à Cayenne ${ }^{84}$. Honoré Michel de Villebois obtient le commissariat de Louisiane.

Les subdélégués montréalais présentent donc de nombreuses caractéristiques urbaines: emploi administratif urbain, mariage tardif, faible investissement rural. Ce sont surtout des allochtones de passage, contrairement à leurs homologues de Québec.

\section{À Québec, des colons magistrats du Roi}

Québec est une ville administrative et marchande. Les subdélégués y sont recrutés parmi les procureurs du Roi et lieutenants généraux successifs. Dans la plupart des subdélégations métropolitaines, les intendants préfèrent être représentés par des magistrats. Ils sont formés aux procédures et familiers du droit et de l'administration. À Québec, ce choix permet aussi d'éviter les querelles de compétence, puisque les subdélégués ont un rôle judiciaire non négligeable. La subdélégation a évidemment de forts caractères urbains. L'intendant change souvent de subdélégués. Ils sont huit à se succéder de 1680 à 1760, en excluant les subdélégués secondaires. La grande majorité d'entre eux sont des colons arrivés de métropole. Ils se sont mariés au Canada. Mais contrairement aux Montréalais, leur installation est pérenne. Lorsqu'ils sont nommés par l'intendant, ils sont déjà en fin de carrière. Ils ont souvent commencé comme secrétaire ou greffier à l'intendance, à la prévôté ou au Conseil supérieur. Certains

82. Marie-Christine-Elisabeth Begon, Lettre au cher fils (Montréal, Boréal, 1994), 189.

83. ANOM, Col., C11A, vol. 120, fol.78-79.

84. Claudine Bougard-Cordier, Inventaire des archives coloniales : articles 1 à 50 (Paris, La Documentation française, 1974), 199, 204 et 205. Jacques François Artur et Marie Polderman, dir., Histoire des colonies françoises de la Guianne (Paris, Ibis rouge, 2002), 488-489. 
ont occupé la place de substitut du procureur avant de devenir procureur du Roi ou lieutenant général.

Installés à Québec, ils investissent avec plus ou moins de succès dans des propriétés urbaines ou rurales. Certains achètent. «Pierre André de Leigne [, selon J.-C. Dubé,] se risqua [ainsi] dans plusieurs entreprises qui, comme à bien d'autres, lui apportèrent plus de soucis que de bons profits ${ }^{85}$ ». Il tente dans les années 1720 d'exploiter au Labrador un domaine de pêche au loup marin. Mais beaucoup acquièrent des terres par leur mariage. Henri Hiché obtient ainsi de l'héritage de l'oncle de sa femme des terres de banlieues qui deviennent, à la suite de ses ventes et concessions, le faubourg Saint-Henry ou faubourg Hiché ${ }^{86}$. Le dernier subdélégué, François Daine, passe une quarantaine d'actes notariés. La moitié de ceux-ci concernent des ventes et achats dans Québec. Il fait construire plusieurs maisons, ce qui lui vaut aujourd'hui d'apparaître sur une plaque au 1080, rue Saint-Jean ${ }^{87}$. L'autre vingtaine concerne les fiefs et seigneuries que lui a procurés son dernier mariage avec Louise Pécaudy de Contrecœur. Propriétaire rural, il n'en reste pas moins urbain et donne la primauté à ses fonctions de juge royal.

En 1760, la conquête bouleverse tout et met en évidence les attaches des subdélégués. François Daine déplore alors «la perte [tant] de [s]es emplois de lieutenant général de la prévosté de Québec, de subdélégué de M. l'intendant et de directeur du Domaine du Roy, que [...] celle de [s]es meubles dont la majeure partie a été pillée par des voleurs et le reste a été consumé par l'incendie arrivé à Québec lors du bombardement ${ }^{88}$ ». À Québec, comme à Montréal, les subdélégués ont construit leur pouvoir sur des charges royales françaises. Ils se préparent alors à quitter le Canada pour la France où les perspectives de carrière sont désormais plus importantes. Les subdélégués secondaires, spécialisés dans la justice, Joseph Perthuis à Québec et Joseph Guiton à Montréal, repartent en France dans les mois qui suivent la défaite ${ }^{89}$. François Daine et le Montréalais Pierre-

85. J.-C. Dubé, "André de Leigne, Pierre», Dictionnaire biographique du Canada en ligne, www.biographi.ca/fr [consulté le 29 décembre 2009].

86. A. Lachance, "Hiché Henry», Dictionnaire biographique du Canada en ligne, www.biographi.ca/fr [consulté le 29 décembre 2009]. Louise Dechêne, «La rente du faubourg Saint-Roch à Québec, 1750-1850 ", Revue d'histoire de l'Amérique française, 34, 4, (1981), 572.

87. Ministère de la Culture du Québec, "Plaque de la maison Louis-Latouche», Répertoire du patrimoine culturel du Québec, www.patrimoine-culturel.gouv.qc.ca/rpcq [consulté le 15 septembre 2015].

88. ANOM, Col. C11A, vol. 105, fol. 239-240.

89. Minutes de Sanguinet dans H. Lafortune, Parchemin..., op. cit. A. Lachance, "Guiton de Monrepos, Jacques Joseph", Dictionnaire biographique du Canada en ligne, www.biographi.ca/fr [consulté le 29 décembre 2009]. 
Michel Martel patientent encore trois ans ${ }^{90}$. Mais ils savent bien qu'ils ne pourront rester si la colonie passe définitivement à l'Angleterre. Tous attendent le traité de Paris et l'officialisation de la cession du Canada pour vendre leurs biens. Comme l'opération a lieu après leur départ, ils font appel à un fondé de procuration. À Québec, François Daine vend deux seigneuries. À Montréal, Pierre-Michel Martel vend une maison ${ }^{91}$. Jusqu'au bout, ils gardent l'espoir de la restitution du Canada à la France. Mais, en 1764, le traité leur impose de partir ou de devenir sujet de Sa Majesté d’Angleterre. Tous les autres subdélégués sont restés au Canada.

\section{À Trois-Rivières, des seigneurs magistrats}

Si Trois-Rivières est une ville, elle n'en reste pas moins une petite ville où l'influence rurale est beaucoup plus forte. Les subdélégués sont des notables locaux investis de fonctions royales. Trois subdélégués se succèdent à TroisRivières de 1688 à 1760. Ils exercent donc en moyenne 24 ans chacun. Ce sont des magistrats, mais avant tout des notables trifluviens. Le premier, Jean Lechasseur, resté célibataire a laissé peu de traces. Par contre, ses successeurs, René Godefroy de Tonnancour et son fils Louis-Joseph sont bien connus. Ils sont nés, se sont mariés et sont décédés à Trois-Rivières. Si le mot n'avait pas une autre connotation en histoire de l'Amérique, nous pourrions écrire que les subdélégués de Trois-Rivières sont des Canadiens. Ils abordent alors la fonction sous un tout autre rapport.

Louis-Joseph Godefroy de Tonnancour a grandi dans sa subdélégation et la connaît par cœur. Surtout, il a complètement hérité de sa situation. En 1731, il succède à son père sexagénaire dans la charge de garde-magasin du Roi. Dès 1734, il exerce la subdélégation à sa place, même si la commission officialisant son remplacement n'est envoyée par l'intendant qu'en 1738, à la mort de René Godefroy ${ }^{92}$. Après le décès, le fils reprend également la place de syndic paroissial. Il ne peut, par contre, succéder à son père dans la charge de lieutenant général. L'intendant juge le procureur Louis-Jean Poulin plus compétent. Louis-Joseph n'obtient donc que l'office de procureur du Roi en $1740^{93}$. Surtout, il hérite de la seigneurie de Tonnancour et de cinq autres fiefs de la région: ceux de Labadie, de Yamaska, de Roquetaille, de Godefroy et de l'île-Marie. Il agit avant tout

\footnotetext{
90. Jacques Mathieu, «Daine, François», Dictionnaire biographique..., op. cit. Dictionnaire biographique du Canada en ligne, www.biographi.ca/fr [consulté le 29 décembre 2009].

91. Minutes de Panet, dans H. Lafortune, Parchemin..., op. cit.

92. BAnQ, Vieux-Montréal, E1, S1, P3078, minutes du notaire Pierre Petit, bobine de microfilm $\mathrm{n}^{\circ} 1467$.

93. ANOM, Col., C11A, vol.120, fol. 267r.
} 
en seigneur et ses activités concernent surtout l'exploitation de ses terres. L'intendant lui reproche d'ailleurs en 1743 de manquer « d'émulation pour s'instruire des lois et de la procédure» et lui refuse à nouveau la lieutenance générale au profit de René-Ovide Hertel de Rouville ${ }^{94}$.

Parmi la soixantaine d'actes notariés conservés en son nom, les deux tiers concernent la gestion de ses propriétés rurales régulièrement concédées ou baillées à ferme. Lorsqu'il reçoit des voyageurs, il leur montre ses terres comme le décrit le mémorialiste Franquet le 29 juillet 1751 . Celui-ci retient surtout de sa visite les «maisons bâties uniformément pour y réfugier des sauvages errans et vagabonds ${ }^{95}$ ». Marié à une ancienne captive des Algonquins, Louis-Joseph Godefroy a appris leur langue. En créant ces abris, il cherche à éviter leurs errances et déviances pour mieux s'en protéger et mieux les contrôler. Mais comme l'explique Louis Franquet, «c'est [aussi] en vue d'augmenter [la] traite [de fourrures avec son magasin] qu'il se constitue en frais pour l'établissement de ce nouveau village ${ }^{96}$ ".

Grand propriétaire foncier, principal commerçant, procureur, syndic de paroisse et subdélégué, Louis-Joseph Godefroy cumule les notoriétés. Son autorité multiforme touche même les Algonquins de la Pointedu-Lac. En 1760, il n'a aucun intérêt à quitter Trois-Rivières. Certes, il perd ses fonctions d'administrateur royal. Mais il conserve ses cinq seigneuries et son négoce. Il reçoit d'ailleurs dès octobre 1760, du gouverneur britannique, la permission d'exercer son activité commerciale. Sa notabilité locale lui permet rapidement de s'intégrer au nouveau régime. Il participe à l'enregistrement du papier du Canada en 1764 et aide ainsi les Anglais à recenser les bons du trésor français pour demander le remboursement à Louis XV. En mars 1769, le gouverneur Guy Carleton le recommande en vain quand il propose d'admettre des francophones au Conseil législatif97. Ses compétences d'ancien procureur royal lui ouvrent tout de même la place de juge de la Cour des prérogatives pour les districts de Trois-Rivières ${ }^{98}$. Le gouvernement anglais a besoin de juges francophones qui connaissent la langue et la coutume de leurs justiciables. Lors de la guerre d'Indépendance et de l'invasion américaine de 1775-1776, Louis-Joseph Godefroy s'engage pour le roi Georges III. Il devient colonel de milice et commissaire de la paix, comme l'indique son acte de sépul-

94. Ibid., vol. 79, fol.58-61v.

95. Louis Franquet, Voyages et mémoires sur le Canada (Québec, A. Coté et Cie, 1889), 23.

96. Id.

97. Frances Caissie, "Godefroy de Tonnancour, Louis-Joseph", Dictionnaire biographique du Canada en ligne, www.biographi.ca/fr [consulté le 29 décembre 2009].

98. Minutes de Maillet dans H. Lafortune, Parchemin..., op. cit. 
ture $^{99}$. Il tente de convaincre les habitants de Trois-Rivières de ne pas rester neutres dans ce conflit entre anglophones et entraîne ses fils à s'engager dans l'armée royale. Il meurt en 1784.

\section{À Détroit et Pabos, des seigneurs et administrateurs}

Le seigneur local est également recruté par l'intendant à Pabos en Gaspésie. Cette subdélégation périphérique à l'aval du fleuve couvre un territoire peu peuplé. Pierre Nadon estime le nombre d'habitants dans la seigneurie à $200^{100}$. Toutefois, sa population varie suivant les saisons. En effet, chaque année des bateaux arrivent de Bayonne, Saint-Malo ou Grandville pour pratiquer la pêche à la morue, au saumon ou à l'anguille. L'intendant nomme donc pour le représenter Georges puis son frère François Lefebvre de Bellefeuille. Seigneurs de Grand-Pabos, ils exercent leur influence de Grande-Rivière à Port-Daniel ${ }^{101}$. Ces seigneurs récoltent toutes les ressources de leur seigneurie côtière. Ils exploitent des pêcheries et prélèvent le onzième des prises de leurs censitaires. Ils louent également des emplacements aux navires de pêcheurs et marchands.

Les Lefebvre de Bellefeuille résident au centre de la baie de Pabos, sur l'île de Beau-Séjour. En 1758, l'Anglais Thomas Bell décrit dans son journal de campagne une maison de « 8 pièces sur un plancher ${ }^{102}$ ». Des fouilles archéologiques ont mis au jour ses restes. On y a découvert la cheminée dont la taille "suggère qu'on y pratiquait une cuisine élaborée» et la salle à manger "qui convient aux rencontres et à la vie sociale d'un seigneur». Pierre Nadon conclut à "un logement qui convien[t] parfaitement aux besoins domestiques sans en faire un symbole d'opulence $»^{103}$. En 1758, les troupes du général britannique James Wolfe détruisent maisons et pêcheries. L'ancien subdélégué quitte alors Pabos pour s'installer à Trois-Rivières dans la famille de son épouse. Il revit alors la situation d'exil de son enfance ${ }^{104}$. Né à Plaisance en 1708, il avait quitté Terre-Neuve après l'invasion britannique et le traité d'Utrecht en 1713. Après une infructueuse tentative de relancer son exploitation, il vend la seigneurie à sir Frederick Haldimand, en 1765, pour en acheter une autre à Trois-

99. Acte $\mathrm{n}^{\circ} 364666$ dans PRDH, op. cit.. et BAC, MG18-H54 1, p. 443-445.

100. Pierre Nadon, La baie du Grand Pabos : une seigneurie gaspésienne en Nouvelle-France au XVIII siècle (Québec, Association des archéologues du Québec, 2004), 24.

101. Id.

102. Ibid., annexe F, 147

103. Ibid, 70 .

104. David Lee, «Lefebvre de Bellefeuille, François» et "Lefebvre de Bellefeuille, Jean-François», Dictionnaire biographique du Canada en ligne, www.biographi.ca/fr [consulté le 29 décembre 2009]. 
Rivières. N'ayant jamais eu d'offices royaux, le changement de couronne n'a aucune conséquence sur ses revenus.

Loin en amont, dans la petite localité de Détroit, résident surtout des soldats, marchands et coureurs des bois qui chassent le castor pour sa fourrure $^{105}$. Monopole royal, cette activité lucrative est encadrée par l'armée et les administrateurs du Domaine. Robert Navarre, notaire et receveur des droits du Domaine d'Occident dispose donc de toute l'autorité appréciable pour la fonction de subdélégué. Il l'exerce en 1743-1752 puis à nouveau en 1754-1759. Entre ces deux périodes, il est remplacé par Jean-Marie Landriève, sieur de Bordes, écrivain principal de la Marine à Détroit. En plus de gérer les dépenses et stocks de l’armée, il contrôle le bon déroulement du marché des fourrures. Les deux hommes sont aussi habitués par leurs fonctions à manier l'écrit et les procédures. Ce sont les meilleurs administrateurs du lieu. Robert Navarre est né en métropole. Il s'installe à Détroit à la fin des années 1720 et s'y marie en 1734. Il acquiert des terres au sud de la rivière en 1747. Il abandonne la subdélégation en $1759^{106}$ mais continue d'exercer comme notaire de Détroit, même après 1763. Il apprend les langues amérindiennes et est peut-être l'auteur du Journal ou dictation d'une conspiration racontant le soulèvement de Pontiac ${ }^{107}$. Il meurt à Détroit en 1791.

Cette variété de profils de subdélégués peut malgré tout se résumer à grands traits par l'opposition entre deux groupes. Le premier rassemble les subdélégués urbains et allochtones de Montréal et Québec qui fondent leur notabilité sur leur place dans l'administration. Le second est celui des subdélégués ruraux, natifs du Canada pour qui la notabilité tient plus à leur terre et à leur réseau social. Ces derniers restent après la Conquête quand les premiers rentrent en métropole.

\section{CONCLUSION}

Les subdélégués de l'intendance canadienne sont-ils des administrateurs urbains ou ruraux? La réponse n'est pas simple. Leurs circonscriptions se basent d'abord sur les trois centres urbains qui structurent le territoire laurentien. Mais les campagnes environnantes se peuplent de plus en plus. Or les prérogatives des subdélégués les concernent en premier lieu. À la ville,

105. Guillaume Teadale, «Old Friends and New Foes: French Settlers and Indians in the Detroit River Border Region", Michigan Historical Review, 38, 2, (2012), 35-62.

106. BAnQ, Québec, E1, S1, P4283.

107. Équipe de la Burton Historical Collection, "Navarre, Robert», Dictionnaire biographique du Canada en ligne, www.biographi.ca/fr [consulté le 29 décembre 2009]. 
les subdélégués sont essentiellement des juges. À la campagne, ils sont responsables de toute la police, de la réfection des chemins au logement des soldats en passant par le recensement. Ils dialoguent alors avec les seigneurs, les capitaines des côtes et communautés de paroisse. Mais ils ne sont pas seulement des représentants de l'intendant. Leur identité est beaucoup plus complexe. Commissaires de la Marine à Montréal, magistrats à Québec, seigneurs à Trois-Rivières et Pabos, les subdélégués sont à l'image de leur chef-lieu. Ils sont recrutés parmi l'élite locale la plus compétente. Or celle-ci est particulièrement variée dans cette province où se côtoient non seulement urbains et ruraux, mais aussi métropolitains et Canadiens.

Loin d'être uniforme, l'intendance canadienne dépasse la seule personne de l'intendant et s'appuie sur des agents très différents d'une circonscription à l'autre. Pourtant, malgré l'étendue du territoire, elle fait le choix d'un nombre restreint de chefs-lieux et donc de diversité. La dualité entre ville et campagne en ressort plus marquée. Cette forte dichotomie entre les circonscriptions et à l'intérieur entre les espaces urbains et ruraux est très particulière au Canada. En France métropolitaine, la diversité des agents et des territoires est moins prégnante. Le cas des autres colonies est moins connu et moins documenté. Il semble toutefois que les subdélégués y soient recrutés parmi les officiers de plume de la Marine. Une étude comparative plus approfondie permettrait de mettre encore plus en relief les pratiques particulières de l'intendance canadienne. 